\title{
Numerical Simulation of Stress and Strain Behavior of Foundation Soil under Compensation Grouting
}

\author{
Alexandra Bestuzheva ${ }^{*}$, and Ivan Chubatov \\ Moscow State University of Civil Engineering, Moscow, Russia
}

\begin{abstract}
To prevent the development of settlements and heave-up of facilities, the compensation grouting technique is used. The article reports the data of numerical simulation of the compensation grouting process as illustrated by the physical experiment performed by Luca Mazini, Ph.D. from La Sapienza Rome University (Rome, Italy). Simulation is performed with the JulyS program developed by the article authors for the ECM. The program implements the finite element method in a three-dimensional approach with Professor L.N.Rasskazov's non-linear "energy" soil model. Different simulation of supplementary volume arrangement in the sandy soil under grouting in the finite element method approach is reviewed. The numerical simulation data are compared with the field experiment.
\end{abstract}

\section{Introduction}

Prevention of limit irregular settlements in waterworks foundation soils is one of the main safety criteria and the governing condition for computation of structures related to the service limit states. However, inevitable irregularity of stress distribution across the structure foot, complicated geological conditions, interaction with the water environment, and seepage flow are often the cause of retaining wall collapse, foundation inclination, uncontrolled settlement of waterworks foundation soils. But the problems of uncontrolled settlements of buildings over the last decades have taken the highest immediacy during the construction of underground traffic infrastructure in the cities. The soil body stabilization technique with compensation grouting technology that provides for the injection of the grout mixtures simultaneously with mining operations with due account for readings of ground and underground transducers to prevent exceeding development of settlements and subsidence of building foundations has been developed first of all to solve the tunneling problems.

Articles intended for "heave-up' effect of the soil surface, foundations of buildings and structures due to injection of supplementary volumes of grout mixtures into the structure foundation soils, thus promoting the subsequent heave-up of the foundation soil, including the structure, are the development of compensation grouting concepts related to soil stabilization [1,2]. The international practice has many examples of implementation of the compensation grouting technology for stabilization of settlements and leveling the structure

*Corresponding author: alex_bestu@mail.ru, 
position. The most famous examples are: leveling the HEP building of the Hessigheim waterworks facility at the Neckar waterway in adjacency to Hessigheim (Germany), compensation of settlement and inclination of the Elizabeth's Tower (Big-Ben) of the Westminster Abbey (London, Great Britain) [3], leveling the building in New Orleans (USA) [4], compensation of settlement of the Bertelsmann office building in Berlin (Germany), etc. Currently, the highest values of compensated settlements under controlled grouting make approximately $170-200 \mathrm{~mm}$. In our country, researches related to leveling the position of Zagorsk PSPP-2 foundation have been performed since 2013. As part of these researches, a successful experiment related to heaving up and assigned leveling of the position of the concrete slab at the test site near Zagorsk PSPP in the process of controlled compensation grouting was performed, for this purpose, soil surface heave- up made approximately $468 \mathrm{~mm}$. The data available on compensation grouting technology allow for approaching this problem with mathematical simulation, the data of this simulation will help to find the best solution for the arrangement of injection of the grout mixtures into the PSPP sand base.

Unfortunately, no known software solutions with an interface designed for solving the equivalent tasks are available at present. Different researchers treated the issues of mathematical simulation of the process of grout injection into the structure foundation soil in different terms: assignment of the finite elements that simulate the injection zone with the supplementary volume strain ratio or thermal expansion coefficient [5].

\section{Methods}

For numerical simulation of the grout injection process under compensation grouting, the proprietary JulyS program for the ECM written in FORTRAN language [6] is used. The program completes stress and strain soil behavior tasks with the finite element method in the three-dimensional approach. A tetrahedron is used as a finite element. The domain is broken into finite elements with the standard program (Gmsh 4.7.1) automatically. The same program allows for visualization of numerical computation data; it is added with the proprietary interpolation modules for this purpose.

To obtain adequate information on the soil ground condition, the non-linear "energy" soil model developed by Professor L.N. Rasskazov [7], where the computational domain is sequentially loaded with the assigned loads and recomputation of the stress and strain soil parameters at each stage show the material hardening effect, is used. Due to lacking experimental data on dilatancy in the sandy soils, the model is used in the simplified form, i.e., excluding dilatancy, creep strain over time to complete the assigned task. In this case, the stress tensor dependency upon strains as per the "energy" model is written as (1):

$$
d \sigma_{i j}=\left[\frac{\delta_{i j} E_{0} d e}{n\left|\sigma_{c p}\right|^{n-1}}\right]+\left[2\left|\sigma_{c p}\right|^{1-n} \cdot\left[f(v) \frac{E_{0}}{n} \exp (B \bar{K}-B)+G_{0} \bar{K}(1-\exp (B \bar{K}-B)]\right] d e_{i j}\right.
$$

where: $\delta_{i j}$ is Kronecker symbol assuming the value $\left\{\begin{array}{l}1, \text { for } i=j \\ 0, \text { for } i \neq j\end{array} ; E_{0}\right.$ and $G_{0}$ is respectively, initial volume and shear strain modules under all-around compression; $f(v)$ is function showing the link of volume and shear strain module at the initial straining moment; $n$ is index; $\sigma_{c p}$ are average soil stresses; $B$ is infinite factor; $\bar{K}$ is hardening parameter: 


$$
\bar{K}=\frac{\kappa_{H}-1}{\kappa_{u}}
$$

The energy condition of strength as per the soil model is written as:

$$
\kappa_{H}=\frac{U_{0}+\int_{L} \sigma d e}{\int_{L} S_{i j} d \varepsilon_{i j}} ;
$$

where: $\kappa_{H}$ is safety factor; $U_{0}$ is preliminary consolidation energy showing the loading history; $\int_{L} \sigma d e$ is energy of shape alteration within the all-around compression area, $\int_{L} S_{i j} d \varepsilon_{i j}$ is energy of shear strains under deviation loading.

Soil model dependency (1) shows the active loading process. For unloading, the Hooke's law is assumed in the following differential form:

$$
d \sigma_{i j}=\delta_{i j} E_{o y} d e+2 G_{o y} d \varepsilon_{i j}
$$

where: $E_{o y}$ and $G_{o y}$ are respectively, volume and shear strain elasticity modules estimated for the unloading legs.

The loading-unloading state condition may be estimated with the total strain energy increment and written as follows:

$$
d \ni=\int_{L} \sigma d e+\int_{L} S_{i j} d \varepsilon_{i j}, \text { where at }\left\{\begin{array}{l}
d \ni \geq 0 \text {-loading } \\
d \ni<0-\text { discharging }
\end{array}\right.
$$

For numerical completion of the tasks with the "energy" model, the initial strain method is used [8]. Completion is made in load increments with the iteration process.

The design program was tested in the elastic approach via the examples with precise analytical solution [9] and compared with the solution on stress and strain behavior of the foundation soil domain under regularly distributed load obtained as per L.N.Rasskazov's "energy" soil model with the local variation method. To assess the adequacy of the design program performance in the soil body expansion domain task approach, the numerical computation data were compared with the field experiment data.

Luca Masini, Ph.D. from Rome La Sapienca University, was engaged in studying soil behavior under compensation grouting. In his article named "Experimental Study of Compensation Injection Technique in Sandy Loam and Loam Soils," he, in cooperation with Cambridge University (Great Britain) [10,11], performed several experiments intended for the study of compensation grouting in the sandy soils as part of the first experimental phase.

Fig.1 shows the used experimental equipment layout. The test chamber (Fig.2) has 850$\mathrm{mm}$ diameter and 400-mm height. Its bottom has an approximately $25-\mathrm{mm}$ thick drain gravel layer covered with the geo-fabric filter. The drainage system is connected with the opened cylinder container via two pairs of holes on the side chamber surfaces and PVC pipes thus, prescribing the experiment conditions in line with the drained arrangement. The 
vertical pressure is applied to the sample via the rubber diaphragm attached to the metal cover around. The cover has five slots for the Linear Variable Differential Transducers (LVDT); each has the through-hole allowing for vertical displacement measurement directly on the sample surface. The grout is supplied into the soil via the horizontal metal pipe with external and internal diameters of $15 \mathrm{~mm}$ and $12.5 \mathrm{~mm}$, respectively, that is entered into the chamber by half of its length and height to ensure grout distribution in the soil strata on all sides from the open pipe end located in the chamber center.

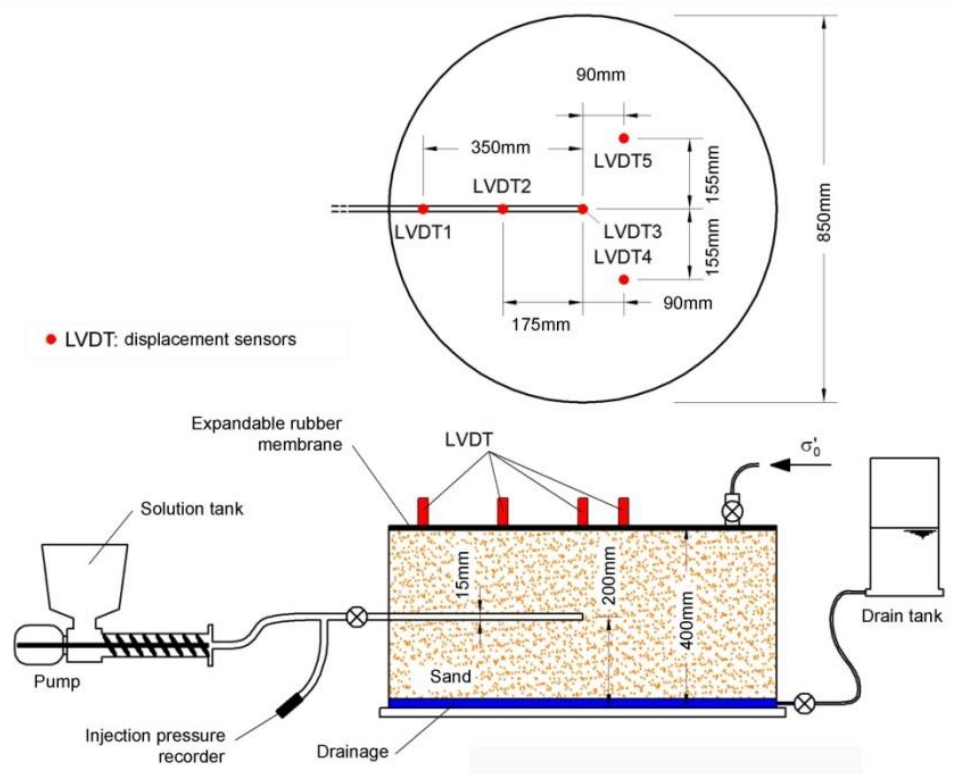

Fig. 1. Used Experimental Equipment Layout

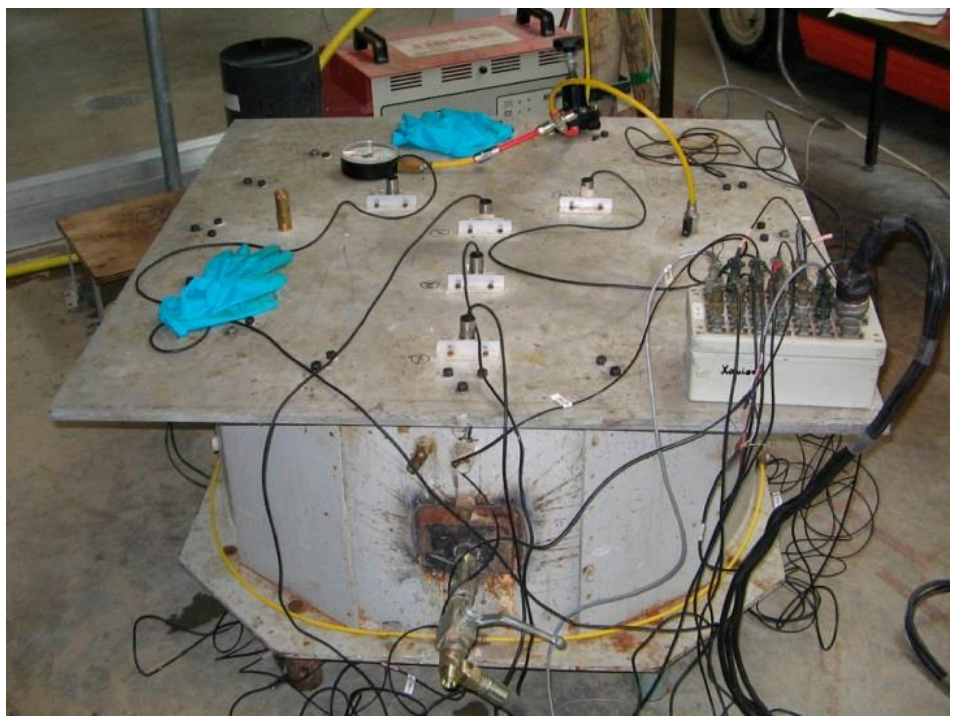

Fig. 2. Cells and Instrumentation Assembled for Experiment 
The soil sample was made from medium-size sand containing $\mathrm{d} 50=0.4 \mathrm{~mm}$ particles. The injected grout with the bentonite additive had the following parameters: (W/G) water/grout ratio - $1.8,(\mathrm{~B} / \mathrm{W})$ bentonite/water ratio -0.08 .

Before grout injection into the soil, the sample surface was applied with $100 \mathrm{kPa}-$ pressure. The total volume of injected grout was made to 1.11 liters. Because of injection, the sample surface was the heaved-up maximum by $4.21 \mathrm{~mm}$; in addition, the heaved-up surface (effective volume) volume made approximately 0.8231 . Hence, grouting efficiency

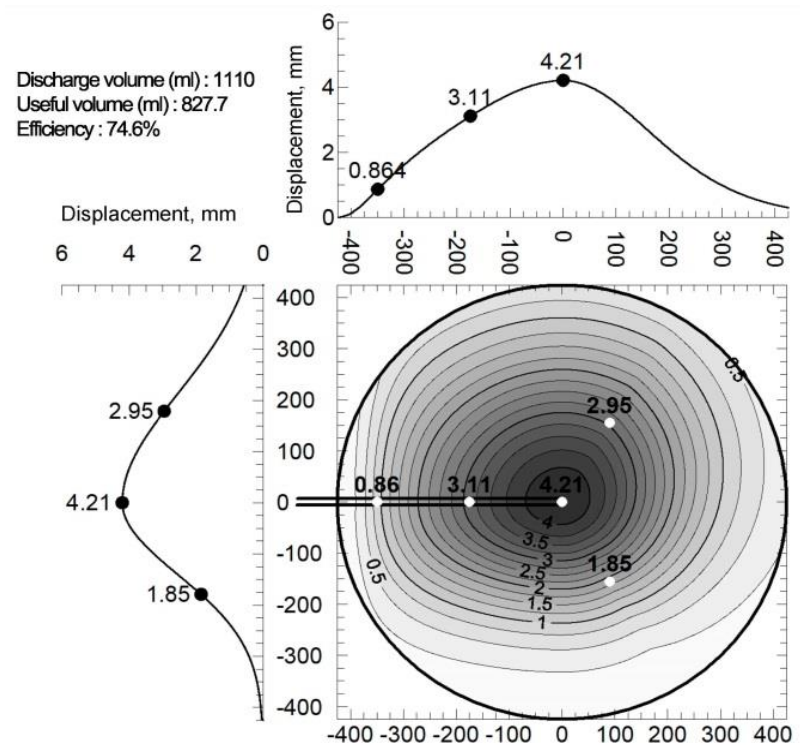

Fig. 3. Surface Displacement Isolines upon Grouting Completion and Longitudinal and Cross Sections Displacement Profiles for Injection Point

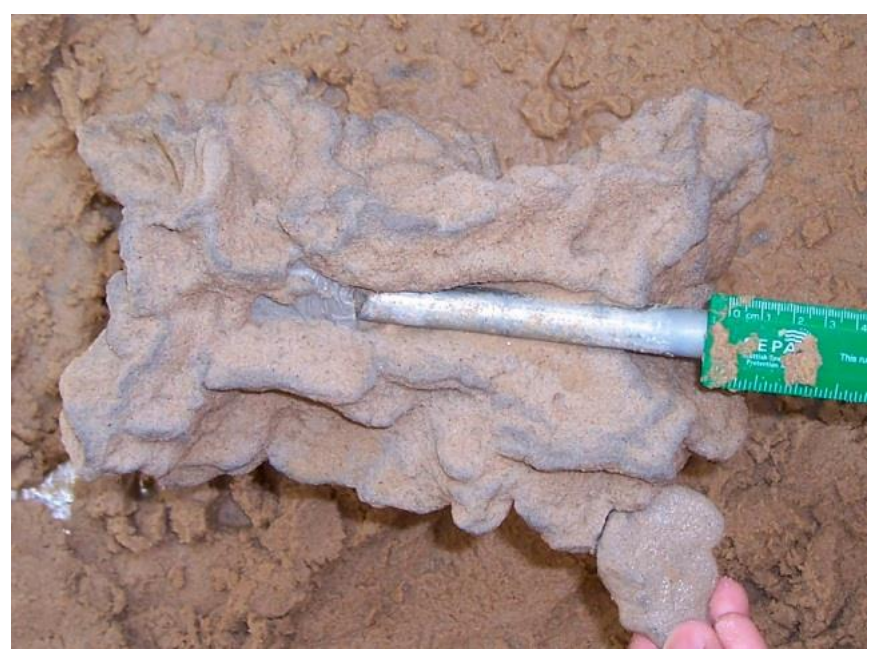

Fig. 4. Obtained Injected Body Top View

in terms of effective volume/supplied grout volume ratio makes $74.6 \%$. Fig.3 shows the experiment data, Fig. 4 shows the obtained injected body. 
Initially, it was assumed that the injected volume would be simulated as the sphere for the numerical simulation. In the process of computation, the total assigned injection volume is broken into portions with due account for the assumed number of grout supply stages. During the computations, the sphere is extended at each stage in line with the injected volume; in addition, should the finite element mesh nodes appear to be inside the sphere, they are assigned movements to ensure their radial entering the assigned sphere border. This process was called "node pulling," Fig.5 shows this process layout.

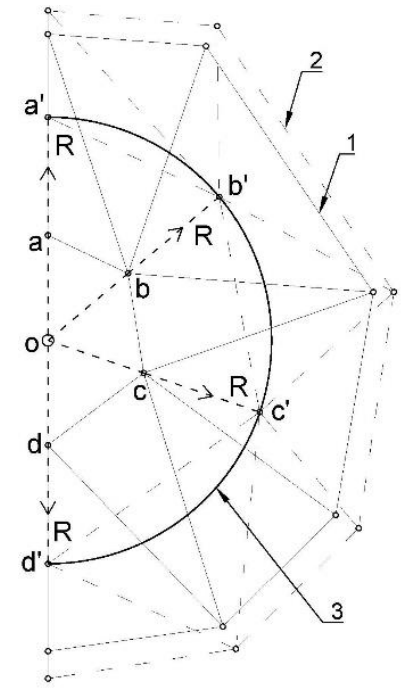

Fig. 5. Layout View of Finite Element Mesh "Node Pulling" to Sphere Border (1 is pre-computation system mesh; 2 is post-computation system mesh; 3 is assigned sphere border; $a, b, c, d$ are system mesh nodes; a', b', c', d' is assigned position of the system mesh nodes, $\mathrm{o}, \mathrm{R}$ is sphere center and radius).

In the process of data analysis of task completion in this approach, it was obtained that tensile stresses appear in the elements at the sphere border. Actually, the soil does not run on tension (exclusive of individual clays and loams). The appearance of tensile stresses evidences the appearance of micro-cracks and material loosening zones that shall inevitably alter the shape of the surface for the injected volume. In addition, it was noted that the grout injected into the sand soil formed not the spherical volume but a more complicated shape (Fig.4). Numerical simulation of this process is very difficult; hence, an issue of searching its approximation to be the most proximal to practicability arose. Two main solutions were selected in the process of searching.

The first - in the structure foundation soil, the vertical stresses always exceed the horizontal ones, in addition, when the grout is injected into the soil at equal pressure on all sides [12], the logical assumption is that inside the soil, the volume shall be shaped extended towards the lower pressure side. In the space, the spheroid (the ellipsoid of revolution) may be assumed as this volume. The horizontal semi-axes in this spheroid in the direction of $\mathrm{a}$ and $\mathrm{b}(\mathrm{x}, \mathrm{z})$ coordinate axes are equal (Fig.6). To simply description of this volume, the horizontal/vertical semi-axes ratio my be assigned. The best spheroid semiaxes ratio was selected via a number of numerical experiments $\left(\frac{b}{a}=\frac{c}{a}=1.5\right)$. 


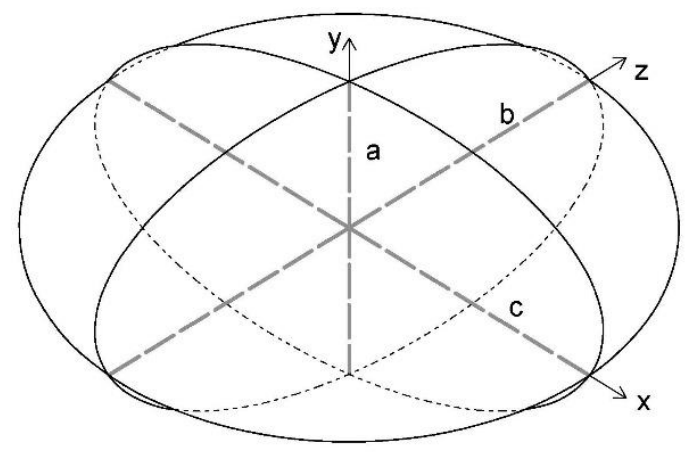

Fig. 6. Spheroid (Ellipsoid of Revolution)

The second - when the finite element mesh was generated, it was decided to provide for the "interior layer" of elements similar to the closed crack at the ends of that, the domain shall be extended into the spheroid shape. The "interior layer" sizes depend upon the sizes of horizontal spheroid axes that are computed at each stage of assigned grout volume injection in line with the equivalent spheroid volume. The spheroid volume was increased with each additional injected portion with the involvement of more new "interior layer" nodes in new volume shaping. For this purpose, the "interior layer" finite element mesh nodes found inside the spheroid "are pulled" to the spheroid surface in its X-direction (Fig.7); as a result, the "interior layer" itself acquires the spheroid shape. The initial reserved size of the "interior layer" was set upon the maximum permissible volume of the injected grout in one injection point (in one sleeve). Before the commencement of grout injection, the "interior layer" elements have adjacent soil properties upon completion of grouting - they are excluded from the computation.

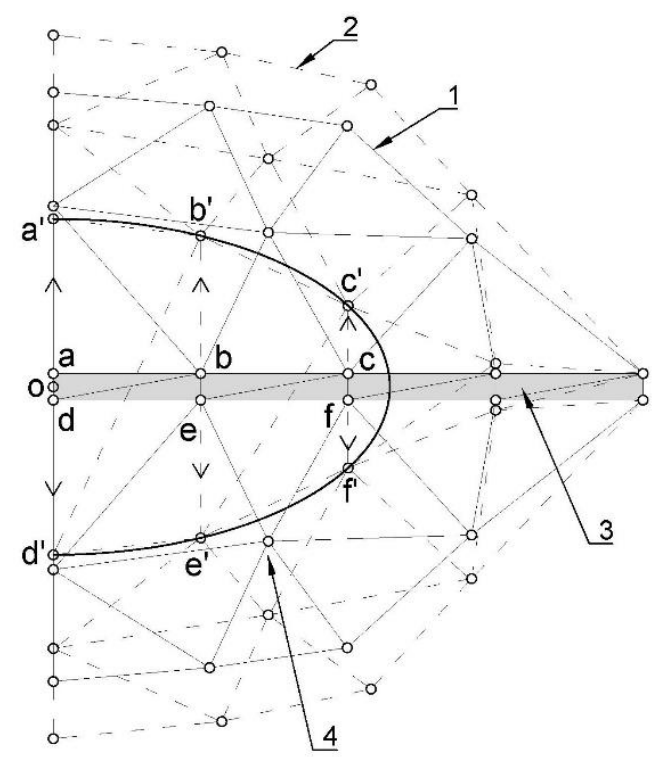

Figure 7. Layout View of "Node Pulling" for "Interior Layer" Elements (1 is pre-computation system mesh; 2 is post-computation system mesh; 3 is "interior layer" of elements; 4 is assigned spheroid border; a, b, c, d, e, f are system mesh nodes; a', b', c', d', e', f' is assigned position of the system mesh nodes, $\mathrm{o}$ is spheroid center) 


\section{Results and Discussion}

For numerical simulation of the physical experiment, the test chamber finite element mesh was restored (Fig.8a). The computation included 12 stages. The first stage included soil computation due to the dead load, the second - computation due to $100 \mathrm{kPa}$ vertical distributed load. Stages 3-12 included simulation of 1.11-1 grout injection into the soil.

a)

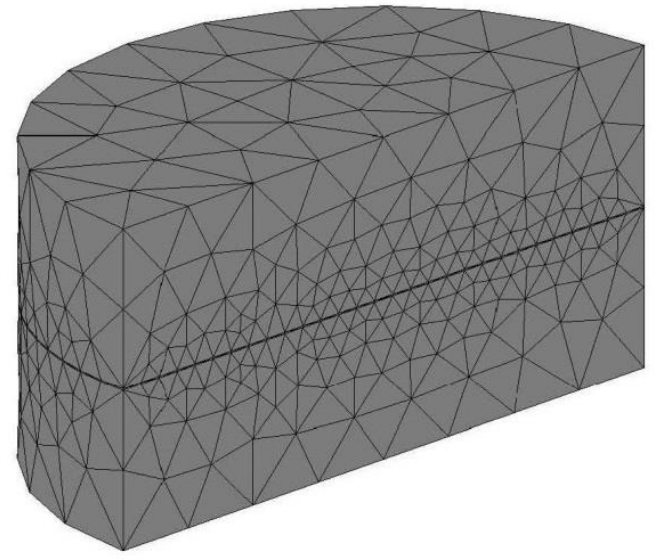

b)

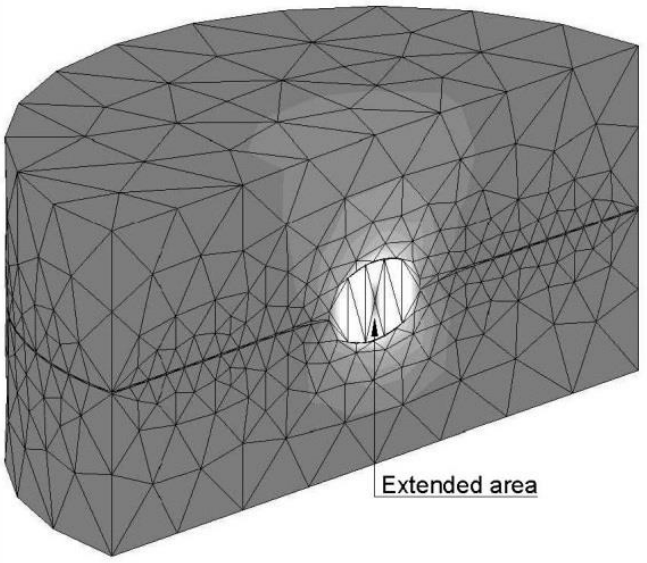

Fig. 8. Test Chamber Half Finite Element Mesh (a is pre-experiment; $b$ is post-injection)

The physical and mechanical soil properties assumed in computation: soil elastic modulus $E=30 \mathrm{MPa}$ (taken from the Building and Facility Design Aid (to SNiP (Construction Codes and Regulations) 2.02.01-83) for medium-size sand); elongation modulus for unloading legs $E_{y}=180 \mathrm{MPa}$; Poisson's ratio $v=0.3$; function value $f(v)=1.5$; degree $n$ $=0.8$; infinite factor $B=10$; specific soil gravity $\gamma=22 \mathrm{kN} / \mathrm{m}^{2}$.

Fig. $8 \mathrm{~b}$ shows the strained finite element mesh following 1.11-1 grouting (stage 12). Maximum heave-up of the sample surface made $4.27 \mathrm{~mm}$ (4.21mm - experiment). Fig. 10 shows isolines of sample surface movements following 1.11-1 grout injection, Fig.11 shows isolines of horizontal section movements. Fig. 12 shows the distribution of average stresses in the horizontal section. As it is seen from the solution results, compression stresses are observed in the entire domain. High compression stress concentration (more than $4000 \mathrm{kN} / \mathrm{m}^{2}$ ) is available on the top and bottom of the extended domain.

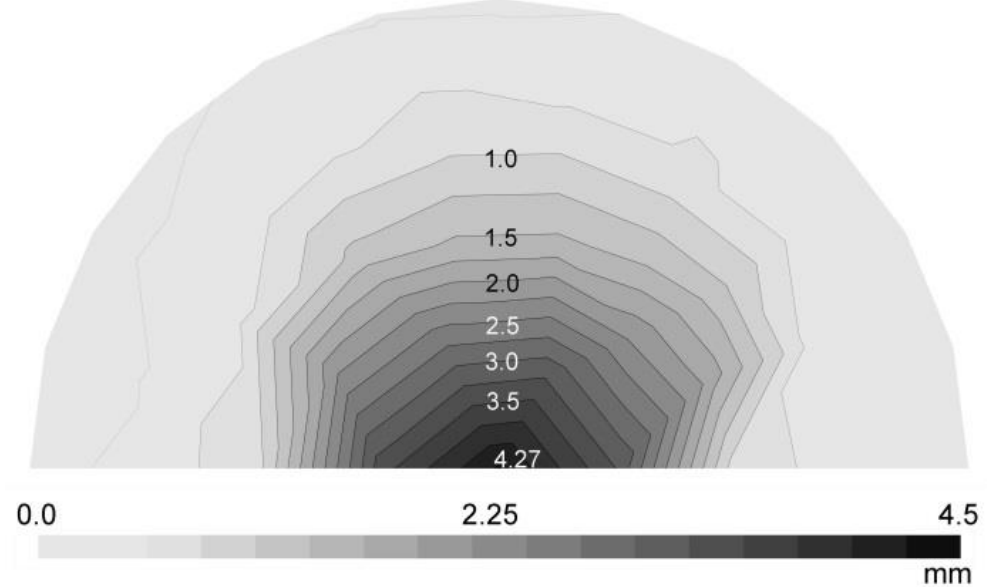


Fig. 10. Sample Surface Movement Isolines Following 1.11-1 Grouting

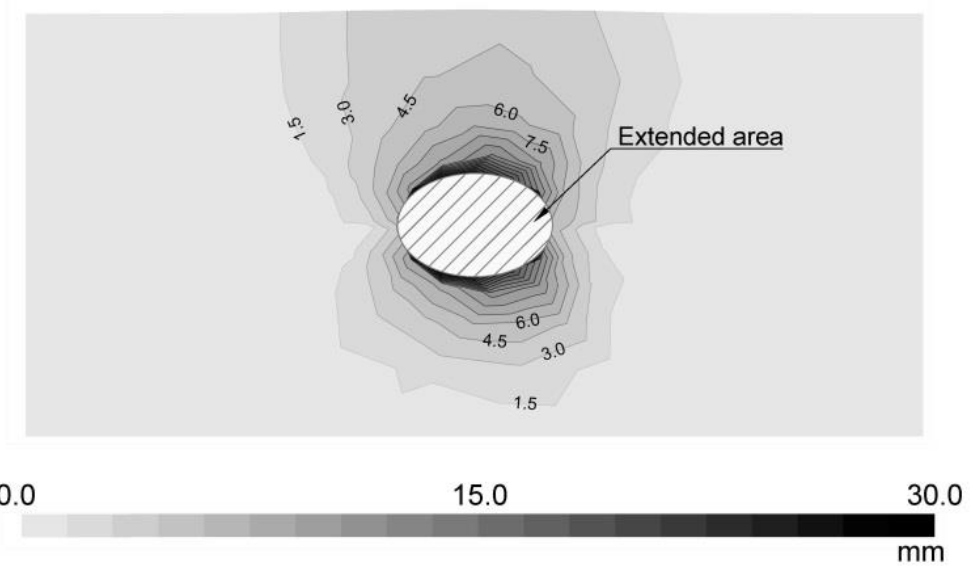

Fig. 11. Horizontal Section Movement Isolines Following 1.11-1 Grouting

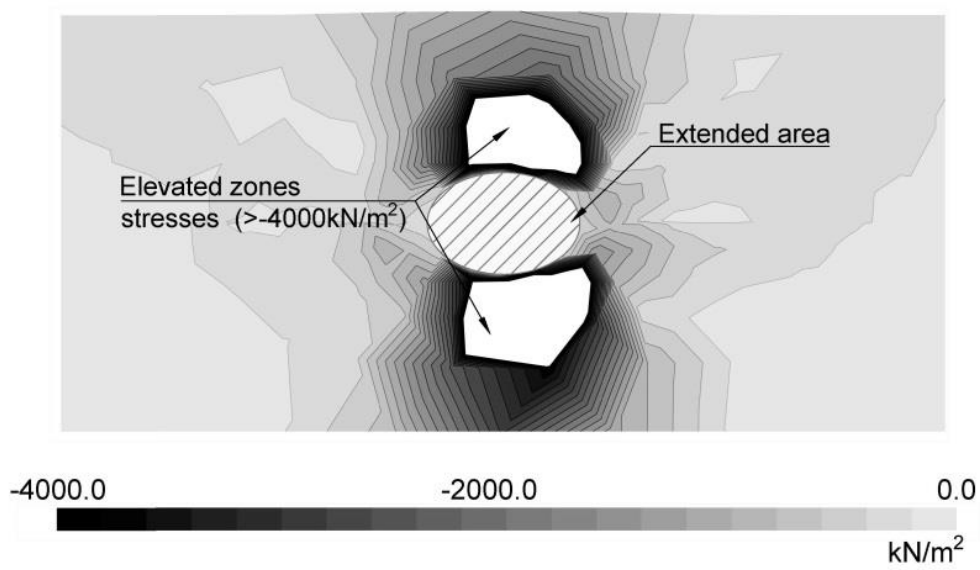

Fig. 12. Horizontal Section Average Stress Isolines Following 11.1-1 Grouting

The sample surface heave-up in the process of numerical computation made $4.27 \mathrm{~mm}$, for the physical experiment $-4.21 \mathrm{~mm}$. The obtained data allow for concluding that the numerical simulation data suitably correspond with the data of grout injection into the sandy soil.

One shall note that a portion of injected grout was probably lost due to water removal into the soil as far as the water/grout ratio made 1.8. Suppose this factor is taken into account for the numerical computation. In that case, the volume of injection domain extension shall be less by the volume of lost "removed" water. The data difference shall not be more than $1.6 \%$, thus showing the high accuracy of the performed numerical research.

\section{Conclusions}

Numerical researches related to simulation of the extending domain in the soil strata have resulted in the development of JulyS computation program allowing for restoration of conditions and re-iteration of the data of performed physical experiment related to grout 
injection into the sandy foundation soil to receive a response as soil surface heave-up. The obtained data error makes max. $1.6 \%$.

The developed mathematical tool for computation of the strain and stress behavior of the soil domain is based upon the common-known L.N.Rasskazov's elastic and plastic soil model focused on the energy condition of strength.

Simulation of the domain of injection and extension around the injection pipe (sleeve) is assumed as the flatten ellipsoid with 1:1.5 ax ratio with increase and extension based upon the "interior layer" elements pre-built into the mesh.

The length of each group of the "interior layer" elements shall be selected with due account for the maximum volume of total injection within this "interior layer" domain.

Numerical experiment efficiency is controlled by lacking tension stresses when the nodes "are pulled" to the sphere border and may be adapted for an individual task in the process of spheroid shape confirmation.

\section{References}

1. M. G. Zertsalov, A. N. Simutin, and A. V. Aleksandrov, Application of Compensation Grouting Technology for Protection of Buildings and Structures Vestn. MGSU 10, 32 (2015)

2. A. Bezuijen, Compensation Grouting in Sand Experiments, Field Experiences and Mechanisms (2010)

3. E. Hamed and M. A. Bradford, Flexural Time-Dependent Cracking and PostCracking Behaviour of FRP Strengthened Concrete Beams (2012)

4. H. Knitsch, Visualization of Relevant Data for Compensation Grouting Tunnel 38 (2008)

5. A. N. Simutin, Technique for Computation of Compensation Grouting Parameters for Control of Strains of Building and Facility Foundation Soils, Moscow State University of Civil Engineering, (2015)

6. A. Bestuzheva and I. Chubatov, in Artic. Collect. Conf. Pap. Second Jt. Constr. Qual. Saf. Cost-Effectiveness Res. Pract. Conf. Pract. Challenges. Prospect. Innov, pp.1213, Moscow, (2019)

7. A. Goldin and L. Rasskazov, Design of Earth Dams. Training Aid (ACB, Moscow, 2001)

8. O. Zenkevich, Finite Element Method in Engineering (Mir, Moscow, 1975)

9. A. Bestuzheva and I. Chubatov, in IOP Conf. Ser. Mater. Sci. Eng. (Institute of Physics Publishing, 2020), p. 072018

10. M. Luca, Studio Sperimentale Della Tecnica Delle Iniezioni Di Compensazione in Terreni Sabbiosi e Limosi, La Sapienza University of Rome, (2010)

11. K. Gafar, K. Soga, A. Bezuijen, M. P. M. Sanders, and A. F. Van Tol, International Society For Soil Mechanics And Geotechnical Engineering Fracturing Of Sand In Compensation Grouting Int. Soc. SOIL Mech. Geotech. Eng. 281 (2009)

12. Z. Ter-Martirosyan, Soils Mechanics (ACB, Moscow, 2009) 